\title{
Strong Radiation of Spin Waves by Core Reversal of a Magnetic Vortex and Their Wave Behaviors in Magnetic Nanowire Waveguides
}

\author{
Sangkook Choi, Ki-Suk Lee, Konstantin Yu. Guslienko, and Sang-Koog Kim* \\ Research Center for Spin Dynamics \& Spin-Wave Devices and Nanospintronics Laboratory, Seoul National University, \\ Seoul 151-744, Republic of Korea
}

(Received 10 February 2006; published 23 February 2007)

\begin{abstract}
We conducted micromagnetic numerical studies on the strong radiation of spin waves (SWs) produced by the magnetic-field-induced reversal of a magnetic vortex core, as well as their wave behaviors in magnetic nanowires. It was found that the radial SWs can be emitted intensively from a vortex core in a circular dot by virtue of localized large torques employed at the core, and then can be injected into a long nanowire via their contact. These SWs exhibit wave characteristics such as propagation, reflection, transmission, interference, and dispersion. These results offer a preview of the generation, delivery, and manipulation of SWs in magnetic elements, which are applicable to information-signal processing in potential SW devices.
\end{abstract}

DOI: 10.1103/PhysRevLett.98.087205

Spin-wave (SW) excitations in bulk or thin-film ferromagnets have long been fundamentally interesting topics in the research area of magnetism [1,2]. In particular, SWs in nanodots, nanowires, and etc., are of revived interest, both theoretically and experimentally, due to progress in submicron (or less) sized sample preparations [3], as well as to the different underlying physics of nanosize elements from that of bulk magnets. Considerable progress in the understanding of spin excitation spectra in confined geometries has been achieved by theoretical approaches, micromagnetic simulations, and sub-ns time-resolving and sub- $\mu \mathrm{m}$ space-resolving measurement techniques. For example, for magnetically saturated elements, not only fundamentals such as the dispersion relation of SWs are derived $[4,5]$, but their propagation, reflection, and tunneling are also verified by various measurement techniques [5-7]. Furthermore, using a magnetic vortex (MV) state in submicron-size elements, the SW modes over the vortex ground state as well as translational vortex motion in the low-frequency regime have been explored by theoretical predictions [8] and experimental verifications [9].

Quite recently, SWs propagating at speeds $\geq 1.0 \mathrm{~km} / \mathrm{s}$ through magnetic nanowire-waveguides (MNWs) have begun to attract considerable attention from the viewpoint of their promising applications for a new paradigm of logic devices [10]. In such devices, information signals can be generated by SW excitations from a local area, and then delivered by propagating SWs through MNWs, and finally manipulated via the superposition. However, the device conception of Ref. [10] assumes only small-amplitude SWs. The signal propagation in SW-based devices is much faster than in newly designed logic devices based on domain-wall propagation [11]. To make SWs applicable practically, it is thus crucially important to excite SWs with sufficiently large amplitudes from a local area like electromagnetic-wave radiation [12], as well as to confirm the fundamental wave behaviors of the SWs propagating along MNWs. In this letter, we report the finding of a
PACS numbers: 75.30.Ds, 75.40.Gb, 75.40.Mg

simple, but novel and controllable method of reliable generation of strong SWs detectable as signals by using a MV core that is a unique ground state in submicron-size magnetic elements. We also report on the wave behaviors of SWs injected into MNWs, such as their propagation, reflection, transmission, interference, and dispersion, as studied by micromagnetic simulations.

Micromagnetic simulation is one of the most optimal tools, and is particularly efficacious in the study of the spin dynamics of dipole-exchange SWs in patterned elements [13], compared with current experimental techniques that are deficient in detecting SWs of wavelengths smaller than $\sim 50 \mathrm{~nm}$, and compared with analytic approaches that are unsuitable for considering exchange and dipolar interactions simultaneously in restricted geometries $[4,14]$. In the micromagnetic approach, the dynamics of the magnetization (M) distribution within a magnet is described by the Landau-Lifshitz-Gilbert (LLG) equation of motion [15], $\partial \mathbf{M} / \partial t=-\gamma\left(\mathbf{M} \times \mathbf{H}_{\mathrm{eff}}\right)+\alpha / M_{s}(\mathbf{M} \times \partial \mathbf{M} / \partial t), \quad M_{s}=$ $|\mathbf{M}|$ with the phenomenological damping constant $\alpha$ and the gyromagnetic ratio $\gamma . \mathbf{M}(\mathbf{x}, t)$ at the position $\mathbf{x}$ precesses around the direction of an effective field $\left(\mathbf{H}_{\text {eff }}\right)$ under effective torque $\left(\mathbf{M} \times \mathbf{H}_{\text {eff }}\right)$ contributed by the exchange, magnetic dipolar, and Zeeman interactions. With advanced computer capability, micromagnetic simulations provide not only sufficient temporal and spatial resolutions but also vectorial information on dipole-exchange SWs.

In the present study [16], we used a model system consisting of a circular shaped, $10 \mathrm{~nm}$ thick Permalloy (Py) dot with a radius of $150 \mathrm{~nm}$ connected to a Py nanowire of width $w=30 \mathrm{~nm}$ and length $l=700 \mathrm{~nm}$, as seen in Fig. 1(a). We used two-dimensional (2D) LLG approach instead of a 3D case [17], because the former is sufficient to have reliable results in such magnetic elements of thickness comparable to the exchange length and allows us to reduce calculation time. The equilibrium configuration of $\mathbf{M}(\mathbf{x})$ was the ground vortex state in the geometry with zero magnetic field as shown in Fig. 1(a) [18], and then was 


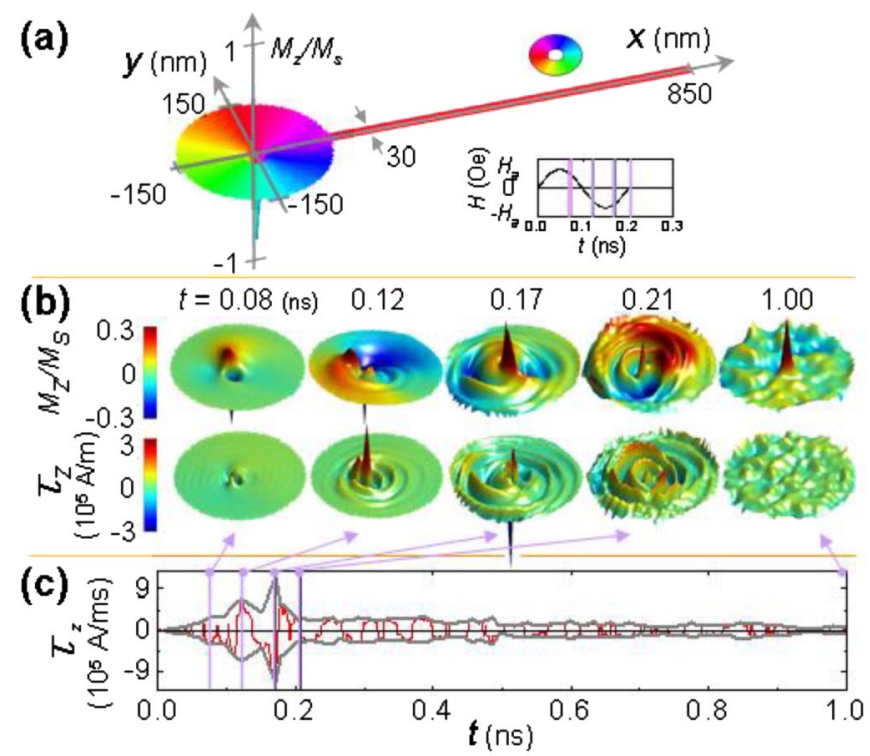

FIG. 1 (color online). (a) Patterned $10 \mathrm{~nm}$ thick Py film model, composed of a circular dot of diameter $300 \mathrm{~nm}$ and a MNW of $30 \mathrm{~nm}$ wide and $700 \mathrm{~nm}$ length. The colors represent the in-plane orientations of the local $\mathbf{M}$, as indicated by the color-coded wheel. The inset shows an applied magnetic field pulse that is sinusoidal with the period of $0.2 \mathrm{~ns}$ and $H_{a}=300$ Oe. (b) Perspective-view images of the spatial distributions of $M_{z} / M_{s}$ and $\tau_{z}$ at the indicated times, $0.08-1.00 \mathrm{~ns}$. (c) Temporal variation of $\tau_{z}$ applied to the core region during its dynamic reversal. The vertical lines indicate the times noted in (b).

perturbed by a sinusoidal field pulse, applied only to the circular dot along the $+y$ direction. The resultant temporal evolution of spin excitations, triggered by a field amplitude $H_{a}$, is displayed in the first row of Fig. 1(b). The temporal variation of the local $\mathbf{M}$ from the initial MV state (at $t=$ $0 \mathrm{~ns}$ ) showed that the radial SWs are generated from the MV core. To understand how the SW modes are excited, the snapshot images of the spatial distribution of the outof-plane $(z)$ components of the local torque, $\tau_{z}=$ $\left(\mathbf{M} / M_{s} \times \mathbf{H}_{\text {eff }}\right) \cdot \hat{\mathbf{e}}_{z}$, and the time dependence of the $\tau_{z}$ at the core only were calculated [the second row of Figs. 1(b) and 1(c), respectively]. During the time duration from $t=0.12$ to $0.17 \mathrm{~ns}$ the MV core is dynamically reversed from the $-z$ to $+z$ direction. Although the exact mechanism of the core reversal is not identified here, it is evident that the core reversal is related to the strong distortions of the profile of the $M_{z}$ of the moving vortex [Fig. 1(b)]. The value of $\tau_{z}$, which is strongly localized in the core region, increases remarkably before reaching its maximum at $t=0.17 \mathrm{~ns}$, whereupon the $\mathrm{SW}$ radiation is also maximized. After the field pulse is turned off, the largest torque is reduced (see images for $t=1.0 \mathrm{~ns}$ ), and hence the excited MV with its reversed core orientation becomes stable (see the movie file [19]). Thus, the MV core plays a crucial role of the radiation source of the SWs.

To elucidate the mechanism by which the radiated SWs are injected from the dot into MNWs as well as their subsequent wave behaviors, the mode spectra in the fre- quency $(f)$ domain were plotted along the $x$ axis at $y=$ $0 \mathrm{~nm}$ for both the circular dot and the MNW [see Fig. 2(a)]. These cases in which the SW radiation is triggered by different values of $H_{a}(100,300$, and $500 \mathrm{Oe}$, represented by rows 1,2 , and 3 , respectively) have some common features. Since the SWs that are able to exist in such a finitesize dot have quantized wavelengths, $\lambda_{n}(n=1,2, \ldots)$ [20], they have various radial nodes depending on their $f$ [see the left column of Fig. 2(a)]. As $n$ increases from 1 to $4, f_{n}$ increases monotonically [20], such that $f_{1}=8, f_{2}=$ $13, f_{3}=15$, and $f_{4}=19 \mathrm{GHz}$, regardless of the magnitude of $H_{a}$. However, the increasing $H_{a}$ leads to the increase of the amplitude of the radiated SWs over the entire $f$ range. The characteristic fast-Fourier transform (FFT) power distributions of the local $M_{z} / M_{s}$ in the circular dot for the quantized values of $f_{n}$ are shown in Fig. 2(b).

The $f$ spectra of the SWs injected from the dot into the MNW show a forbidden band for $f<f_{p \text {,Py }}=14 \mathrm{GHz}$, the value of which corresponds to the minimum $f$ of the SW modes that are allowed to propagate through the Py nanowire of the given dimension. Those SWs of $n=1$ and 2 excited within the circular dot $\left(f<f_{p \text {,Py }}=14 \mathrm{GHz}\right)$ are not allowed to have real wave vector values to satisfy the dispersion of SWs existing within the Py MNW. Consequently, they do not propagate through the MNW, but, on the contrary, are reflected backwards into the circular dot. Thus, standing SWs with various radial nodes are formed within the dot, as mentioned above. By contrast, SWs of $n=3,4,5, \ldots$ (i.e., $f>f_{p \text {,Py }}=14 \mathrm{GHz}$ ) propagate well through the entire length of the MNW and approach its end. Then, they are reflected backwards, thereby producing a definite pattern of standing waves due to the superposition of the forward and backward traveling SWs, as is evidenced by the number of nodes of $\lambda / 2$ length along the $x$ axis near the right-side end.

Figure 2(c) illustrates the dispersion relations, $f$ vs $k_{x}$, the longitudinal component of the wave vector $\boldsymbol{\kappa}=$ $\left(k_{x}, k_{y}\right)$, for the SWs traveling within the MNW. For each of the different $H_{a}$ values, three distinct modes are observed, as indicated by the three different-colored lines marked in Fig. 2(c). In the region of $f>f_{p, \text { Py }}=14 \mathrm{GHz}$, two different concave-up curves (marked as the black and red lines) appear, indicating that there are strong dispersions between $f$ and $k_{x}$ for the SWs traveling along the long axis of the MNW. The cases $k_{x}>0$ and $k_{x}<0$ correspond to the SWs traveling forward and backward, respectively. $k_{x}$ can be considered to be quasicontinuous due to the sufficiently long MNW. The primary concave branch (the black line) above $f_{p \text {,Py }}=14 \mathrm{GHz}$ corresponds to the SWs traveling primarily along the $x$ axis, that is, having the minimal value of the $k_{y}$. The value of $k_{y}$ is quantized due to the pinning on the stripe lateral edges $y= \pm w / 2$ [21]. The corresponding SW-mode profiles, eigenfrequencies, and effective pinning were calculated for thin stripes $(L / w \ll 1)$ in Ref. [21]. Our case, with thickness $L=10 \mathrm{~nm}$ and $w=30 \mathrm{~nm}$, is more compli- 
(a)

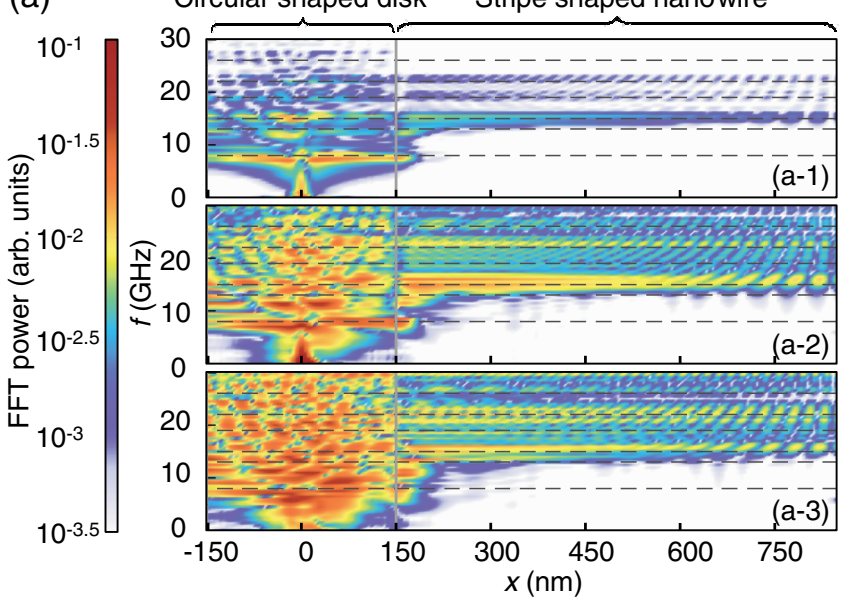

(c)

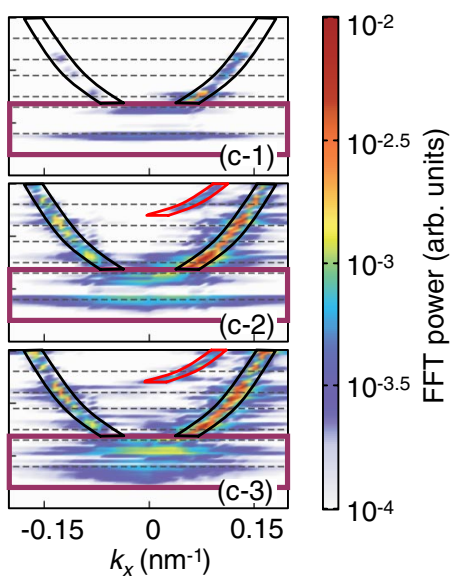

(b)

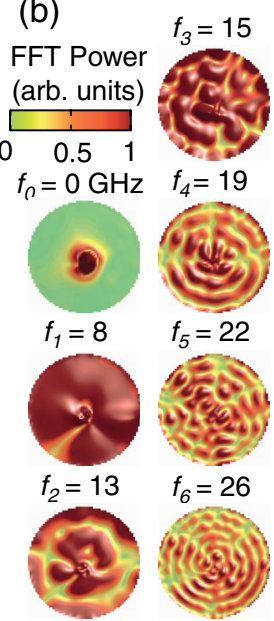

FIG. 2 (color online). (a) $f$ spectral images along the $x$ axis with $y=0$, obtained from the FFT of the $M_{z} / M_{s}$ oscillations in the circular dot (left column) and the MNW (right column). (b) The FFT power distributions of the local $M_{z} / M_{s}$ for $f_{n}=8,13,15,19,22$, and $26 \mathrm{GHz}$. (c) Dispersion relations of the SWs traveling only within the MNW $(150<x<850 \mathrm{~nm})$ along the $x$ axis at $y=0$. The first, second, and third rows correspond to $H_{a}=100,300$, and $500 \mathrm{Oe}$, respectively. The gray-colored dashed lines stand for each value of $f_{n}$. The black-, red-, and purple-colored solid lines are marked to display the three different regions mentioned in the text.

cated because the exchange interaction cannot be ignored and $L / w \sim 1$. The approximate dispersion relation [4] is

$$
\begin{aligned}
\omega_{m}^{2}\left(k_{x}\right)= & {\left[\omega_{H}+\omega_{M} L_{e}^{2} \kappa_{m}^{2}+\omega_{M} f\left(\kappa_{m} L\right) k_{y, m}^{2} / \kappa_{m}^{2}\right] } \\
& \times\left\{\omega_{H}+\omega_{M} L_{e}^{2} \kappa_{m}^{2}+\omega_{M}\left[1-f\left(\kappa_{m} L\right)\right]\right\}
\end{aligned}
$$

with $f(x)=1-[1-\exp (-x)] / x$,

where $\omega_{H}=\gamma H_{\mathrm{in}}, \omega_{M}=4 \pi \gamma M_{s}, L_{e}=\left(A / 2 \pi M_{s}^{2}\right)^{1 / 2}$ is the exchange length, and $\kappa_{m}^{2}=k_{x}^{2}+k_{y, m}^{2}$ is the in-plane wave vector. The internal field $H_{\text {in }}$ is equal to zero everywhere in the stripe except in the region $\sim L$ near its edges.

The Eq. (1) is an increasing function of $k_{x}$ for sufficiently large values of $k_{x} \geq 0.05 \mathrm{~nm}^{-1}$ [see Fig. 2(c)]. For the given value of $k_{x}$, the value of $\omega_{m}$ is quantized and can be approximately described according to the discrete values of the $k_{y, m}$, where $m=1,2$, etc. To estimate the forbidden $f$ gap in Figs. 2(a) and 2(c), we inputted $\omega_{H}=$ $0, k_{x}=0$ (neglecting the localized modes along the $x$ axis), and $m=1$ into Eq. (1), leading to

$$
\omega_{1}^{2}(0)=\omega_{M}^{2}\left[L_{e}^{2} k_{y, 1}^{2}+f\left(k_{y, 1} L\right)\right]\left[L_{e}^{2} k_{y, 1}^{2}+1-f\left(k_{y, 1} L\right)\right] .
$$

It is evident from Eq. (2) that the gap $f, \omega_{1}(0) \propto M_{s}$, has an exchange-dipolar origin and is not equal to zero, due to $k_{y, 1} \neq 0$. The estimation of $k_{y 1}$ on the basis of the pinning parameter [21] yields the value $k_{y, 1}=0.065 \mathrm{~nm}^{-1}$ or $k_{y, 1} w \approx 1.94$. The calculation by Eq. (2), $f_{p, \text { Py }}=$ $\omega_{1}(0) / 2 \pi \approx 17.2 \mathrm{GHz}$, is in reasonably good agreement with micromagnetic simulations $\left(f_{p, \text { Py }}=14 \mathrm{GHz}\right)$. The exchange interaction reduces the pinning parameter for MNWs with $L \sim L_{e}$ [21]. Based on the above analytical approach, we can interpret the dispersion relations in the following manner. Each minor branch (the red line), whether, according to $H_{a}$, it is strong or extremely weak, is associated with the SWs traveling dominatingly along the $y$ axis in the MNW, because it is of finite width $w=$ $30 \mathrm{~nm}$. The exchange energy contributes considerably to the transverse mode $\left(k_{x}=0\right)$ frequencies, which are rapidly increasing with increasing quantized $k_{y, m}$. Hence, the secondary SW branch $(m=2)$ has essentially higher $f$ s than the primary SW branch $(m=1)$. In contrast to these concave branches, flat branches (the purple line) prevailing

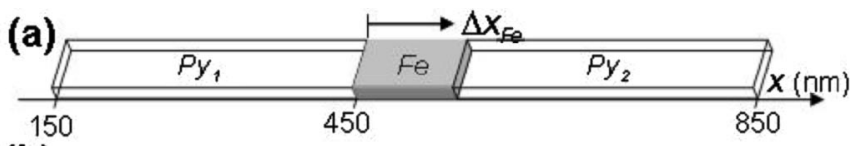

(b)

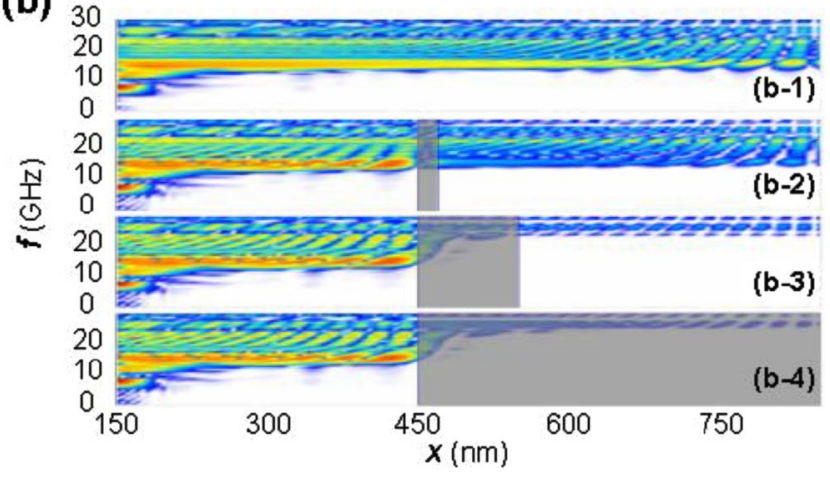

FIG. 3 (color online). (a) Heterogeneous structure of the $\mathrm{Py} / \mathrm{Fe} / \mathrm{Py} \mathrm{MNW}$ with a total length of $700 \mathrm{~nm}$, where an $\mathrm{Fe}$ segment with different lengths, $\Delta x_{\mathrm{Fe}}=0,20,100$, and $400 \mathrm{~nm}$, is inserted from the position $x=450 \mathrm{~nm}$. (b) The $f$ power spectra along the $x$ axis at $y=0$ in the different waveguides. The first, second, third, and last rows correspond to $\Delta x_{\mathrm{Fe}}=0$, 20, 100, and $400 \mathrm{~nm}$, respectively. 
over a wide range of $k_{x}$ appear in the region of $f<f_{p \text {,Py }}=$ $14 \mathrm{GHz}$, which corresponds to strongly spatially localized modes at the nanowire-end edge. The ripple contrast (which is extremely weak, we note the logarithm scale) at the bottom of Fig. 2(c) is a fundamental property of SWs in restricted geometry. There are few low- $f$ modes in the excitation spectrum of magnetic wires. The corresponding eigenmodes are localized near the edges and cannot be adequately described by a single $k_{x}$ value (see Refs. $[5,6]$ ). Although all the three branches are commonly observed, their FFT powers vary significantly with $H_{a}$, in accordance with the allowed and dominant SW modes.

Next, we examine the wave behavior of SWs traveling through $\mathrm{Py}_{1}(300 \mathrm{~nm}) / \mathrm{Fe}\left(\Delta x_{\mathrm{Fe}} \mathrm{nm}\right) / \mathrm{Py}_{2}\left(400-\Delta x_{\mathrm{Fe}} \mathrm{nm}\right)$ [22] [see Fig. 3(a)], where $\Delta x_{\mathrm{Fe}}$ is the length of the Fe segment, such as $0,20,100$, and $400 \mathrm{~nm}$. The Fe and Py segments are saturated along the $x$ direction, thereby locally inducing a higher $f$ gap in the $\mathrm{Fe}$ than in the $\mathrm{Py}_{1}$ segment. Figure 3(b) shows the resultant SW power spectra, with each $\Delta x_{\mathrm{Fe}}$, along the $x$ axis at $y=0 \mathrm{~nm}$ in the heterostructures. The $f$ gap of $\mathrm{Py}_{1}$ reaches $f_{p, \mathrm{Py}}=$ $14 \mathrm{GHz}$. In contrast, as $\Delta x_{\mathrm{Fe}}$ increases, the $f$ gap in the Fe becomes higher, ultimately reaching $f_{p, \mathrm{Fe}}=24 \mathrm{GHz}$, which is the minimum $f$ of allowable SW modes propagating along the Fe segment [23]. We note that the ratio of the $f$ gaps for $\mathrm{Fe}$ and Py MNWs is 1.71 , and is approximately equal to the ratio of $M_{s, \mathrm{Fe}} / M_{s, \mathrm{Py}}=1.98$, in agreement with approximate equation (2).

The SW power spectra in $\mathrm{Py}_{2}$ for the case of $f_{p, \mathrm{Py}}<$ $f<f_{p, \mathrm{Fe}}$ are quite different for each $\Delta x_{\mathrm{Fe}}$. Since the $f$ gap of $\mathrm{Fe}$ is higher than that of Py, the SWs arriving at the $\mathrm{Py}_{1} / \mathrm{Fe}$ interface are partially transmitted through it and partially reflected backwards with a reduced amplitude, as seen for $\Delta x_{\mathrm{Fe}}=20 \mathrm{~nm}$. When $\Delta x_{\mathrm{Fe}}$ is sufficiently long, the forward traveling SWs are totally attenuated within the Fe segment and, thus, entirely reflected backward from the $\mathrm{Py}_{1} / \mathrm{Fe}$ interface [Figs. 3(b-3) and 3(b-4)]. This reflection becomes strong with decreasing $f$ in the region of $f_{p, \text { Py }}<$ $f<f_{p, \mathrm{Fe}}$. By contrast, in the cases of $f>f_{p, \mathrm{Fe}}=$ $24 \mathrm{GHz}$, the SWs are transmitted intensively through the $\mathrm{Fe}$ segment and then propagate well inside $\mathrm{Py}_{2}$ or the entire $\mathrm{Fe}$ region, independently of the value of $\Delta x_{\mathrm{Fe}}$. Consequently, we note that the lower- $f$ SWs existing in Py can be filtered out by the sufficiently long Fe segment.

In conclusion, we (i) demonstrate, for the fist time, the radiation behavior of SWs produced from a MV core associated with its reversal, (ii) show the injection behavior of SWs from a circular dot into a MNW, and (iii) verify how to control the frequencies of SWs using MNW heterostructures. All of the three points are crucially important for future technological applications to SW devices, including logic devices, with regard to the generation, delivery, and control of information signals, respectively. These are also the key points in understanding the fundamental wave properties of SWs in restricted geometry.
This work was supported by Creative Research Initiatives (ReC-SDSW) of MOST/KOSEF.

*Corresponding author.

Electronic address: sangkoog@snu.ac.kr

[1] L. R. Walker, in Spin Waves and Other Magnetic Modes, edited by G.T. Rado and H. Suhl, Magnetism Vol. 1 (Academic, New York, 1963).

[2] B. Heinrich and J.A.C. Bland, Ultrathin Magnetic Structures I (Springer, Berlin, 1994).

[3] Spin Dynamics in Confined Magnetic Structures, edited by B. Hillebrands and K. Ounadjela, Topics in Applied Physics Vol. 83 (Springer, Berlin, 2002).

[4] K. Y. Guslienko and A. N. Slavin, J. Appl. Phys. 87, 6337 (2000); K. Yu. Guslienko et al., Phys. Rev. B 68, 024422 (2003).

[5] C. Bayer et al., Phys. Rev. B 72, 064427 (2005).

[6] J. Jorzick et al., Phys. Rev. Lett. 88, 047204 (2002).

[7] S. O. Demokritov et al., Phys. Rev. Lett. 93, 047201 (2004); J. P. Park et al., Phys. Rev. Lett. 89, 277201 (2002).

[8] K. Yu. Guslienko et al., J. Appl. Phys. 91, 8037 (2002); B. A. Ivanov and C. E. Zaspel, Phys. Rev. Lett. 94, 027205 (2005).

[9] S.-B. Choe et al., Science 304, 420 (2004); J. P. Park and P. A. Crowell, Phys. Rev. Lett. 95, 167201 (2005); M. Buess et al., Phys. Rev. B 71, 104415 (2005).

[10] R. Hertel, W. Wulfhekel, and J. Kirschner, Phys. Rev. Lett. 93, 257202 (2004).

[11] D. A. Allwood et al., Science 296, 2003 (2002).

[12] K.-S. Lee, S. Choi, and S.-K. Kim, Appl. Phys. Lett. 87, 192502 (2005).

[13] M. Grimsditch et al., Phys. Rev. B 69, 174428 (2004).

[14] R. Arias and D. L. Mills, Phys. Rev. B 70, 094414 (2004).

[15] L. D. Landau and E. M. Lifshitz, Phys. Z. Sowjetunion 8, 153 (1935); T. L. Gilbert, Phys. Rev. 100, 1243 (1955).

[16] We used the OOMMF code. See http://math.nist.gov/ oommf. The micromagnetic parameters used for Py were as follows: the exchange stiffness $A=13 \mathrm{pJ} / \mathrm{m}$, the saturation magnetization $M_{s}=8.6 \times 10^{5} \mathrm{~A} / \mathrm{m}$, and zero magnetic anisotropy constant. The cell size is $5 \times$ $5 \times 10 \mathrm{~nm}^{3}$ and $\alpha=0.01$.

[17] M. Buess et al., Phys. Rev. Lett. 94, 127205 (2005); F. Boust and N. Vukadinovic, Phys. Rev. B 70, 172408 (2004).

[18] N. Dao, S. L. Whittenburg, and R. P. Cowburn, J. Appl. Phys. 90, 5235 (2001).

[19] See EPAPS Document No. E-PRLTAO-98-001710 for two movie files. For more information on EPAPS, see http:// www.aip.org/pubservs/epaps.html.

[20] K. Yu. Guslienko et al., Phys. Rev. B 71, 144407 (2005).

[21] K. Yu. Guslienko et al., Phys. Rev. B 66, 132402 (2002); K. Yu. Guslienko and A. N. Slavin, Phys. Rev. B 72, 014463 (2005).

[22] We used the parameters $A=21 \mathrm{pJ} / \mathrm{m}$ and $M_{s}=1.7 \times$ $10^{6} \mathrm{~A} / \mathrm{m}$ for the Fe segment. For the value of $A$ at the $\mathrm{Fe} /$ Py interface, their harmonic mean of $16 \mathrm{pJ} / \mathrm{m}$ was used.

[23] The value of $f_{p, \mathrm{Fe}}=24 \mathrm{GHz}$ is determined from the dispersion relation of SWs propagating in the same geometry as shown in Fig. 1(a), but made of Fe, with a perturbation by the field pulse with $H_{a}=300 \mathrm{Oe}$. 